\title{
A Study on High Performance Embedded Systems with Multiple Processors
}

\author{
Prakash H T ${ }^{1}$, Dr. Srinivas $\mathbf{M}^{2}$ \\ Technical Assistant, Department of Technical Education, Board of Technical Examination, Bangalore, India ${ }^{1}$ \\ Principal and Director Research and Development, St. Mary’s Group of Institutions, Hyderabad, India ${ }^{2}$
}

\begin{abstract}
The progress made in growing more propelled compilers for embedded systems, programming of embedded elite computing systems in light of Digital Signal Processors (DSPs) is as yet an exceedingly talented manual assignment. This is valid for single-processor systems, and significantly more for embedded systems in light of numerous DSPs. Compilers regularly neglect to improve existing DSP codes written in C because of the utilized programming style. Parallelization is hampered by the complex various address space memory design, which can be found in most business multi-DSP arrangements. This postulation builds up a coordinated advancement and parallelization methodology that can manage low-level $\mathrm{C}$ codes and creates enhanced parallel code for homogeneous multi-DSP architecture with disseminated physical memory and numerous sensible address spaces. In an initial step, low-level programming phrases are distinguished and recouped. This enables the use of abnormal state code and information changes surely understood in the field of scientific computing Iterative criticism driven scan for "good" change groupings is being researched. A novel approach to parallelization in view of a bound together information and circle change structure is displayed and assessed. Execution improvement is accomplished through abuse of data locality from one viewpoint, and usage of DSP-particular building components, for example, Direct Memory Access (DMA) exchanges then again. A novel parallelization structure containing data distribution and territory improvements has been concocted and experimentally assessed against significant DSP benchmarks. Vital commitments to the improvement of novel gathering methods for superior embedded systems in view of single processors and in addition on multiple processors have been made. It is likely that we will see more research here as future Systems-On-Chip will involve bigger quantities of heterogeneous processors with non-standard memory architectures, which will challenge existing compiler technology.
\end{abstract}

Keywords: High Performance, Embedded Systems, Multiple Processors, progress, programming, Digital Signal Processors, DSPs, memory design, architecture, memory, information, improvement, DMA, technology.

\section{INTRODUCTION}

High Performance Computing is not the elite area of computational science. Rather, high computational power is required in many devices, which are not worked with the essential objective of furnishing their clients with a computer of any sort, however to offer an administration in which an intense computer plays a focal part. Medicinal imaging is a case of the utilization of such a High Performance Embedded System [1]. As signs from an X-beam or magnetoreverberation gadget come in at a high rate, they are handled by a computer to furnish the radiologist with a perception appropriate for further determination. Different cases incorporate radar and sonar processing, speech combination and acknowledgment, and a wide scope of uses in the fields of mixed media and telecommunications [2-4].

In this study, embedded systems in view of Digital Signal Processors (DSPs) are examined as one particular case of the various framework designs being used today [5]. Ongoing computerized flag handling requires high-performance processors because of the strict planning imperatives forced by the unstable way of signs. Digital Signal Processors (DSPs) are omnipresent and progressively critical in the telecommunications and electronics industry [6-8]. The business' interest for brief time to-market, high computational execution, low power utilization and adaptability over the life expectancy of their gadgets - e.g. to adjust to new norms, to add new components or to right bugs of prior variants - settle on programmable DSPs the most loved decision for some new electronic designs.

From the imperatives set by the DSP application area emerge a few (halfway fundamentally unrelated) necessities for signal processors particular to those of general purpose processors. DSPs must have the capacity to convey enough computational power to adapt to requesting applications like picture and video handling while meeting further limitations, for example, minimal effort and low power [9-11]. As a result, DSPs are generally exceedingly particular and adjusted to their particular application area, however famously hard to program. DSP applications have inspecting rates that fluctuate by more than twelve requests of size. Climate estimating on the lower end of the recurrence scale has examining rates of around $1 / 1000 \mathrm{~Hz}$, however uses exceptionally complex algorithms, while requesting radar applications require inspecting rates over a gigahertz, yet apply moderately simple algorithms. Both extremes have in 
like manner that they depend on elite processing frameworks, potentially in view of DSPs, to meet the planning requirements forced on them. With the present condition of processor technology, it is as yet unrealistic to convey the required compute power for a few applications with only a solitary DSP, yet the consolidated energy of a few DSPs is required. Sadly, such multi-DSP frameworks are significantly harder to program than a solitary DSP.

Efficient execution of DSP applications is basic for some embedded systems. Improving C compilers to a great extent concentrate on code era and booking, which, with their developing development, are giving unavoidable losses. In this study another approach in light of abnormal state source-to-source changes is exactly assessed. While program execution as of now advantages from the use of individual changes, the maximum capacity is just acknowledged when a few changes are joined. Be that as it may, the recognizable proof of a fruitful change succession is a non-insignificant errand and static methodologies frequently flop because of the mind boggling connection between abnormal state changes, the backend compiler and the objective design. Besides, static examination is typically restricted by the way that compiler producers once in a while record the low-level changes connected by their compilers. Iterative investigation of the change space, then again, does not expect any learning of the backend compiler and is yet ready to discover successful change groupings. This is accomplished by substituting change and execution organizes and choosing the best alternative a short time later.

Digital signal processing and media processing are execution basic applications for embedded processors. This interest for execution has prompted the advancement of particular models, with application programs hand-coded in gathering. All the more as of late as the cost of building up an inserted framework winds up plainly overwhelmed by algorithm and programming improvement, there has been a move towards the utilization of abnormal state programming dialects, specifically C. As in different regions of processing, programming in $\mathrm{C}$ is a great deal less tedious than hand-coded constructing agent, yet this comes at the cost of a less proficient usage when contrasted with hand-coded approaches.

\author{
Objectives of the study: \\ 1. To study the High Performance Embedded Systems. \\ 2. To study the High Performance Digital Signal Processing. \\ 3. To study High-Level Transformations for Single-DSP Performance Optimizations. \\ 4. To study the Parallelization for Multi-DSP. \\ 5. To study the Localizations and Bulk Data Transfers in Embedded Systems.
}

\title{
1.4- Hypotheses of the study:
}

H1: There is relationship between High Performance Embedded Systems and Digital Signal Processing.

H2: A key aspect of the High-Level Transformations for Single-DSP Performance Optimizations.

\section{REVIEW OF LITERATURE}

It was around the years 2003 to 2005 that an emotional change grabbed the semiconductor industry and the makers of processors. The expanding of computing performance in processors, based on essentially spoiling the clock recurrence, could at no time in the future be holded. Every one of the years prior to the clock frequency could be consistently expanded by improvements achieved both on innovation and on structural side. Scaling of the technology processes, prompting littler channel lengths and shorter exchanging times in the gadgets, and measures like guideline levelparallelism and out-of-request preparing, prompting high fill rates in the processor pipelines, were the underwriters to meet Moore's law However, beneath the $90 \mathrm{~nm}$ scale, the static power dispersal from spillage current outperforms dynamic power dissemination from circuit exchanging. Starting now and into the foreseeable future, the power thickness must be restricted, and as an outcome the expansion of clock recurrence came about to stagnation. In the meantime architecture improvements by removing parallelism out of serial direction streams were totally depleted. Hit rates of over $99 \%$ in branch forecast couldn't be enhanced further on without sensible exertion for extra rationale hardware and chip area in the control unit of the processor. The appropriate response of the business to that advancement, keeping in mind the end goal to at present meet Moore's law, was the moving to genuine parallelism by multiplying the number of processors on one chip pass on. This was the introduction of the multi-center range. The advantages of multi-center computing, to meet Moore's law and to restrain the power thickness in the meantime, in any event right now this announcement holds, are additionally the reason that parallel registering in light of multi-center processors is in progress to catch increasingly likewise the universe of embedded processing.

Gupta et al. (2000): An address optimization in light of a succession of source-to-source changes is appeared and assessed in Gupta et al. (2000). This enhancement depends on express exhibit gets to and does not work with pointerbased programs. Here the pointer-conversion algorithm can be connected as a preliminary stage that empowers the further streamlining. In spite of the fact that going for DSP applications the exploratory outcomes originated from universally useful CPUs. It is not in the least evident if the change stretches out to DSPs as the creators guarantee, and a show of this is still outstanding. 
Qian et al. (2002): evaluate the effectiveness of abnormal state loop transformations, for example, unroll-and-stick and loop unrolling with regards to grouped VLIW architectures, e.g. the Texas Instruments TMS320C6x. In light of measurements obtained from software pipelining, they compute loop unroll considers and unroll-and-stick adds up to apply the loop under assessment. Experimental results are gathered on one simulated (URM) and one genuine engineering (TI TMS320C64x), in view of an arrangement of 119 loops from a DSP benchmark set. Speedups in the scope of 1.4 to 1.7 were accomplished on various machine arrangements. Loop transformations in this study are executed in Memorial, a source-to-source FORTRAN transformer in view of the System. The benchmarks, in any case, are composed in $\mathrm{C}$ and were physically meant FORTRAN to empower change. The changed codes were then physically made an interpretation of back to C. It is uncertain whether this translation process could be mechanized, specifically, regarding pointers and other low-level develops in the C source. Moreover, loop unrolling in the TI backend compiler was turned off for the experimental evaluation. In this manner, it ends up plainly not clear how well their approach performs in contrast with TI's execution of loop unrolling.

Falk et al. (2003): In two novel controls stream changes pertinent to address overwhelmed multimedia applications are created. Going for the disposal of data transfer and capacity overheads presented by past change stages, Loop Nest splitting and Ring Buffer Replacement are in part coordinated in the SUIF compiler. Loop nest part is a speculation of conventional loop exchanging, which makes it conceivable to manage circle subordinate if-explanations. Ring cushion substitution tries to supplant round supports of little size with an arrangement of scalar factors, which cause bring down tending to overhead. The effectiveness of the changes is assessed against seven different platforms and two selected benchmarks. Normal picks up in execution time are in the range from $40.2 \%$ to $87.7 \%$ with normal code measure overhead in the vicinity of $21.1 \%$ and $100.9 \%$. Although successful on the picked benchmarks, both changes give off an impression of being very particular. Moreover, circle settle part depends on an unpredictable and costly genetic algorithm.

Kulkarni et al. (2003): Kulkarni et al. (2003) investigate the issue of discovering effective optimization stage groupings and propose an intuitive client guided way to deal with the stage ordering problem. As another option to manual streamlining, an automated approach in view of a genetic algorithm to scan for the most effective enhancement succession in view of indicated wellness criteria is assessed. Among the advancement stages considered are both highlevel and low-level changes, for example, circle invariant code motion and enlist portion, individually. Unrolling, tiling, and other high-level transformations usually utilized in numerical codes are, be that as it may, not considered. Extra measures to counteract obstruction of high-level and low-level changes are necessary, e.g. to avert enroll al-area before enlistment variable acknowledgment has been performed. Results are exhibited for an important embedded benchmark suite (MiBench), yet the target architecture (SPARC) does not really speak to the qualities of normal embedded processors. While the client guided way to deal with streamlining is an extremely effective tool for master clients, the normal client won't not have the capacity to completely misuse its potential. The programmed hereditary search algorithm hardly outflanks the settled stage arrange baseline approach now and again, however requires at least 100 eras to achieve this. This study exhibits an extremely fascinating way to deal with input coordinated iterative arrangement and streamlining for embedded systems. Tragically, empirical data is gathered for a broadly useful CPU and not effortlessly transferable to embedded architectures.

Su et al. (1999): Software pipelining is a guideline planning technique for loops, in which consequent cycles are covered to accomplish higher ILP. Su et al. (1999) exhibit a source-to-source loop transformation in view of software pipelining. Despite the fact that $\mathrm{C}$ does not have the capacity to express parallel explanations, their approach goes for reordering $\mathrm{C}$ articulations such that manufacturers' backend compilers can create more productive code. They assess their technique on eight DSP kernels for single target architecture. Average speedups of $16 \%$ are achieved. The fairly little arrangement of benchmarks and the limitation to single target architecture limits the helpfulness of this study. It is not clear how well their techniques perform on different architectures or with more propelled compilers. Besides, previous work has demonstrated that the advantages from source-level software pipelining primarily begin from the expanded flexibility in booking guidelines of the bigger circle body and can without much of a stretch be accomplished (and even outflanked) with considerably less demanding to actualize loop unrolling.

High Performance Digital Signal Processing: Digital Signal Processors (DSPs) are omnipresent and progressively vital in the broadcast communications and hardware industry. The business' interest for brief time to-market, high computational execution, low power utilization and adaptability over the life expectancy of their gadgets - e.g. to adjust to new guidelines, to add new elements or to right bugs of prior forms - settle on programmable DSPs the top choices decision for some new electronic plans. For instance, the business magazine EE Times reports of impressive growth rates forecasts. From the limitations set by the DSP application space emerge a few (halfway fundamentally unrelated) prerequisites for signal processors unmistakable to those of broadly useful processors. DSPs must have the capacity to convey enough computational energy to adapt to requesting applications like image and video processing while meeting further imperatives, for example, ease and low power. As a result, DSPs are normally very particular and adjusted to their particular application space, yet famously hard to program. DSPs find application in a wide scope of various flag handling situations, which are portrayed by their algorithm complexity and transcendent examining rates. 
A review of these properties for various applications is given in figure 1. DSP applications have testing rates that change by more than twelve requests of greatness. Climate gauging on the lower end of the recurrence scale has testing rates of around $1 / 1000 \mathrm{~Hz}$, however uses exceedingly complex algorithms, while requesting radar applications require examining rates over a gigahertz, yet apply generally simple algorithms. Both extremes have in like manner that they depend on elite computing systems, potentially in view of DSPs, to meet the planning imperatives forced on them. With the present condition of processor technology, it is as yet unrealistic to convey the required compute power for some applications with only a single DSP; however the consolidated energy of several DSPs is required. Sadly, such multiDSP frameworks are significantly harder to program than a single DSP.

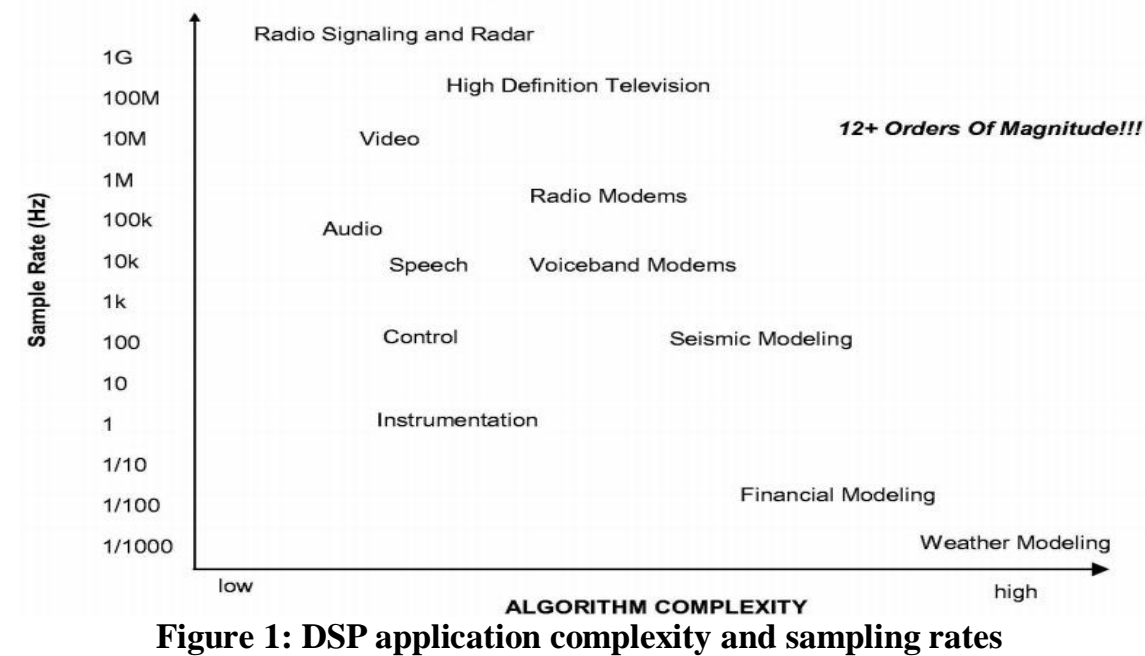

Parallelism in DSP Applications: DSP and media algorithms are frequently very redundant as approaching information streams are consistently prepared This normality recommends that DSP and multimedia applications contain more elevated amounts of parallelism than general purpose applications, potentially at various granularities. Figure 2 demonstrates the intrinsic parallelism of three classes of workloads (broadly useful, DSP, video). DSP and video codes contain bigger measures of exploitable parallelism than broadly useful codes, with video codes containing the most parallelism. This reality not just rearranges the work of naturally parallelizing compilers, however more essentially it gives the premise to bigger execution benefits as per Amdahl's Law. While broadly useful codes can just experience hypothetical speedups of up to 10 because of parallel execution, DSP and sight and sound codes are liable to more than request of greatness higher performance improvements. In the past, DSP software was for the most part made out of little bits and software development in low level computing construct was satisfactory. Like different fields of computing, code complexity in the DSP area started to increment and application advancement utilizing abnormal state dialects, for example, $\mathrm{C}$ turned into the standard. Late DSP applications require ten thousand or more lines of C code. Issues abusing the parallelism in DSP codes emerge from this utilization of $\mathrm{C}$ as the commanding abnormal state dialect for DSP programming. $\mathrm{C}$ is especially hard to examine because of the huge degrees of opportunity given to the programmer. Far more atrocious, C allows a low-level; equipment arranged programming style that is habitually utilized by embedded systems programmers to physically tune their codes for better performance. Without exact examinations, be that as it may, accomplishment in recognition abuse of program parallelism is extremely constrained.

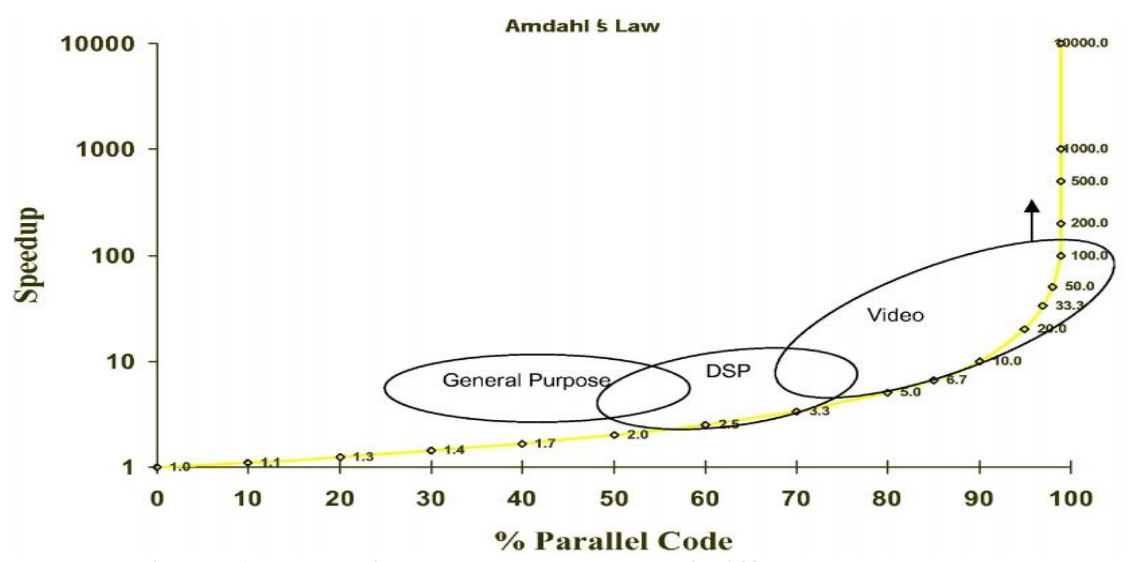

Figure 2: Potential parallel speedup of different workloads 
Against this foundation, advancing and in addition parallelizing compilers must figure out how to adapt to eccentricities of the $\mathrm{C}$ programming language and the dominating programming style so as to be successful.

Parallelism in DSP Architectures: DSP manufacturers' reaction to the expanded interest for computational energy of their gadgets was the appropriation of the Very Large Instruction Word (VLIW) worldview to offer bigger measures of Instruction-Level Parallelism (ILP) in their processors. This approach is exceptionally engaging as enhanced semiconductor producing innovation takes into consideration the coordination of more useful units on the same chip while keeping up the same consecutive abnormal state programming model. Be that as it may, it introduces the compilers for these structures with the issues of distinguishing at the same time executable directions and of developing minimized and efficient schedules Figure 3 shows the execution prerequisites of average DSP applications. While for most current end-client media transmission applications a solitary DSP suffices, more register escalated applications in the media transmission framework, sight and sound and discourse handling spaces require more PC power than an individual DSP can convey. To oblige these requesting applications, arrangements to consolidate individual DSPs to a multi-DSP were taken by their makers. By the by, multiprocessor capacities of most commercial DSPs are exceptionally limited because of cost when contrasted and bigger standard parallel PC frameworks. Once more, makers take after their plan theory to actualize just the most habitually used usefulness in equipment. This negligible equipment bolster has critical results for the plan of parallel DSP software. Composing parallel code for a multi-DSP target is as yet a very talented, manual errand with related expenses because of individual power, expanded time-tomarket and reduced reliability.

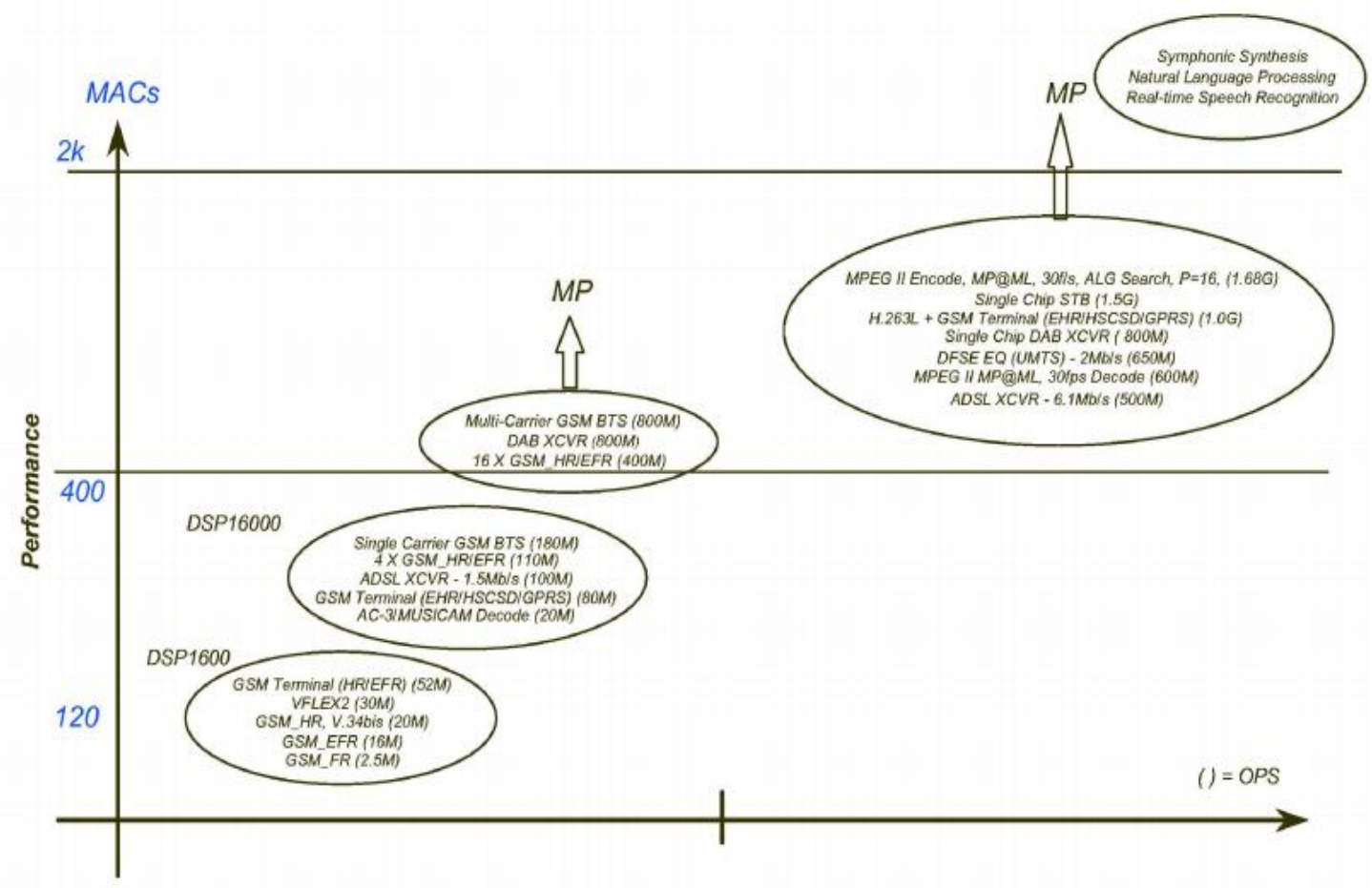

Figure 3: Application performance requirements

High-performance embedded processing: Modern embedded markets call for high thickness computing ability, making it is hard to utilize only one microchip to meet capacity prerequisites of elite embedded systems. Multiple processors, including broadly useful embedded microprocessors, digital signal processors (DSPs), ASICs and FPGA equipment quickening agents, are frequently utilized as a part of these embedded systems. Not all processors in an embedded device have similar qualities and they are deviated. Heterogeneous multiprocessors present challenges in both equipment and software designs. The review addresses the issues of supporting parallelization in asymmetric multiprocessor (AMP) condition from both equipment and software sides, including reserve soundness, semaphore and embedded software programming. With the improvement of silicon technologies, embedded chips turn out to be all the more effective and have more thick computing ability. Embedded processors replace broadly useful PC processors on account of their ease as well as due to their low influence utilization, rich usefulness and high dependability. Embedded microprocessors have been utilized extensively in consumer electronics, (for example, sight and sound players and gaming gadgets) and specialized gadgets, (for example, mobile phones and individual computerized partners). Nonetheless, individuals seek after for elite will never stop. Numerous embedded devices still call for high computing 
ability, making it is hard to utilize only one microprocessor to fulfill the functionalities. For example, one progressed 200 MIPS ARM processor is not sufficiently capable to translate MPEG4 or H.264 video motions in a set top box. In numerous embedded systems, more than one processor is utilized to accomplish superior, various errands keep running in parallel [1]. Typically, the frameworks have three solutions:

1) Designing software for a few symmetric broadly useful embedded microprocessors (SMP) [2] [3] running in parallel;

2) One universally useful embedded microprocessor in addition to programmable hardware accelerators (FPGAs), application specific integrated circuits (ASICs), and digital signal processors (DSPs). The broadly useful participates with the particular equipment to improve performance;

3) Asymmetric multiprocessors (AMP) [4] [5], mixing multi-center chip, DSPs, FPGAs and ASICs.

Asymmetric Multi-Processor Architecture: With the development of silicon technology, an ever increasing number of transistors can be incorporated in a solitary chip, multicore processors are technique trend both in embedded and PC markets. Multi-center processors have points of interest in both superior and low-control utilization. On the off chance that the processing cores of a processor are indistinguishable, the processor is known as a symmetric multi-processor (SMP) ARM11 MP Core TM is a SMP [9] [10]. An ARM MP Core processor can be arranged to have 1-4 processor cores. Figure 4 outlined the design of an ARM11 MP Core processor with 4 processor cores. The processor cores in this processor are indistinguishable, which make it simple for a solitary OS to timetable assignments inside centers in balance. As described before, SMPs for the most part bolster just unadulterated software solutions, in which, "hard" continuous can't be ensured. The application-particular processors, such as FPGAs, DSPs and ASICs, are incorporated to meet most pessimistic scenario idleness. Application-specific circuits are additionally required in embedded systems to improve the execution and power proficiency of some important program 'parts'. In one our initial study, the attributes of embedded programs were broke down [8]. Embedded processors for the most part arrangement just with a settled number of applications, and among these applications, CPU occupation rates are very lopsided.

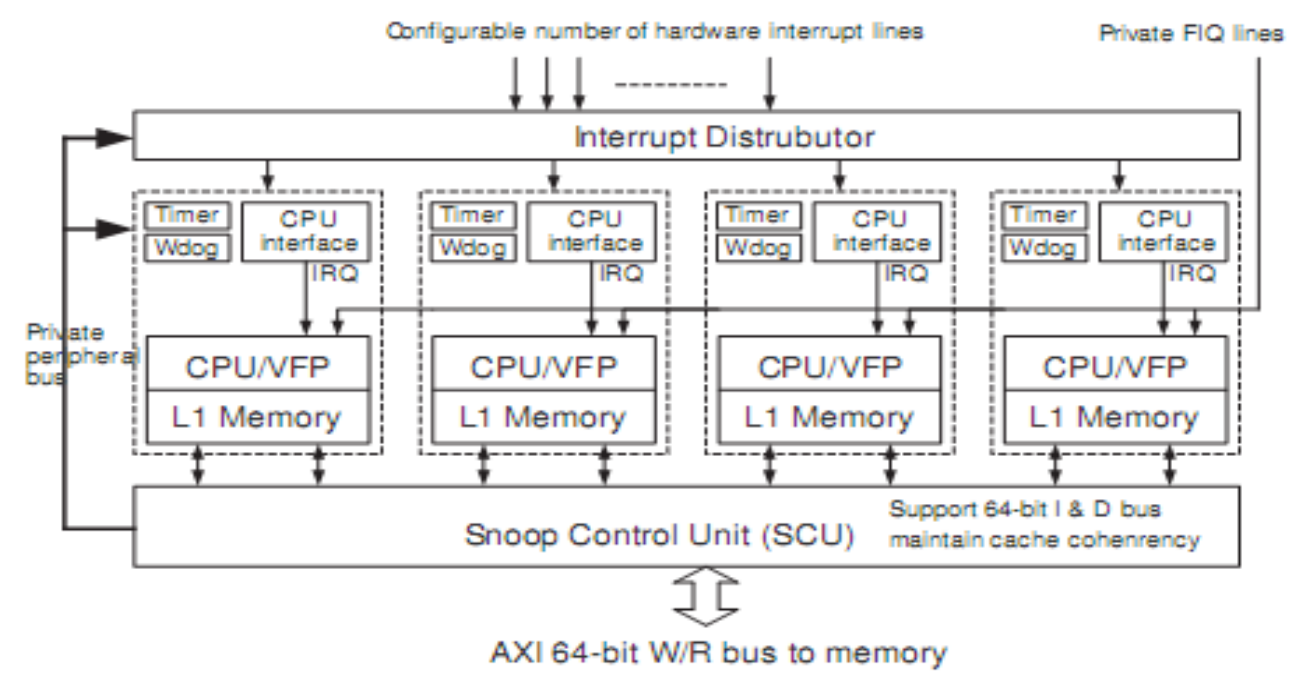

Figure 4- The Architecture of a symmetric multi-processor

Embedded processors may invest the majority of their execution energy in executing just a couple circles of a couple programs. Thus, few vital program pieces (program fragments which might be little circles or capacity calls) have the best effect on the achievement of an embedded processor. In addition, since the execution and power-proficiency of these essential bits are basic for general execution and power utilization, the bits are regularly hand-enhanced, yet application-particular circuits can significantly enhance the productivity of execution than unadulterated software solutions. Particularly outlined equipment can significantly improve the execution and power-effectiveness for these important kernels, and along these lines, DSPs, FPGAs and ASICs are typically chosen by implanted framework fashioners. The contribution of these application-particular processors makes the various processors deviated. The design of an asymmetric multi-processor (AMP) is outlined in Figure 5.

AMP architecture incorporates a few identical microprocessors, a few different microprocessors, and some applicationparticular circuits. In this manner, an AMP framework is a blend of SMP and AMP. SMP and AMP have their own particular memory area. In any case, they have to communicate and collaborate, a common memory area is essential. To beat the bottleneck of memory and processor, a little nearby store is regularly put between processors and main 
memory. An information lucidness component must be utilized to guarantee the accuracy and consistency of information. AMP architecture presents challenges for embedded hardware plan and parallel software programming.

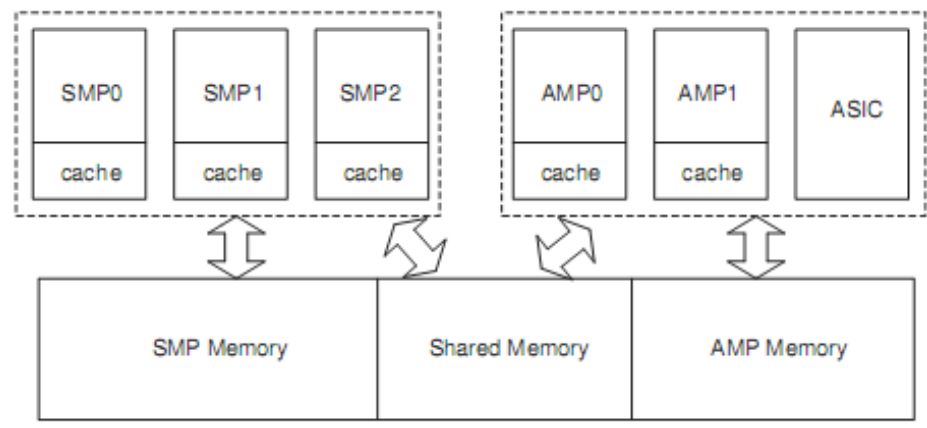

Figure 5- The Architecture of an asymmetric multi-processor

Principles of embedded multi-core processors:

Multi-core processors in embedded systems: In this sub study, we indicate quickly a sort of transformative improvement including a stepwise mix of processor principles, known from standard processors, into embedded processors. The last stride of this improvement procedure is the presentation of multi-center innovation in embedded processors. Perhaps the most trademark highlights of ARM processors are their small chip bite the dust sizes and their low power necessities. Both elements are obviously of high significance for applications in embedded environments. ARM is a result of ARM Inc., Cambridge, England. ARM fills in as a fables organization that implies they don't manufacture chips; also they outline microchips and microcontrollers and pitch these plans under permit to different organizations. Embedded ARM architectures can be found in numerous handheld and shopper items, as e.g. in Apple's iPod and i Phone gadgets. In this manner, ARM processors are presumably not just a standout amongst the most broadly utilized processors in embedded designs yet a standout amongst the most overall utilized processors at all. The main ARM processor, denoted as ARM1, was a 32-bit RISC (Reduced Instruction Set Computer) processor. It emerged in 1985 as result of the organization Acorn, which outlined the primary business RISC processor, the Acorn RISC Machine (ARM), as a coprocessor for a computer used at British Broadcasting Corporation (BBC). The ARM1 was extended towards an incorporated memory management unit, a design and I/O processor unit and an upgraded guideline set like duplicate and swap directions and discharged as ARM2 around the same time. After four years, in 1989, the processor was furnished with a bound together information and direction level one (L1) store as ARM3. It took after the support of 32-bit addresses and the incorporation of a gliding point unit in the ARM6, the combination of further components as System-on-Chip (SoC) in the ARM6, and static branch expectation units, more profound pipeline stages and upgraded DSP (Digital Signal Processing) offices. The outline of the ARM6 was additionally the main result of another organization, shaped by Acorn, VLSI and Apple Computer. In 2009 ARM discharged with the Cortex-A5 MP Core processor their first multi-core processor expected for utilization in cell phones. The aim was to give one of the littlest and most power-productive multi-core processor to accomplish both the execution that is required in cell phones and to offer low expenses for cheap chip fabricating. Precisely like the ARM11 MP Core, another multi-center processor from ARM, it can be designed as a gadget containing up to 4 centers on one processor die.

Brief overview of selected embedded multi-core architectures: The ARM Cortex A9processor means the second era of ARM's multi-center processor technology. It was likewise expected for preparing broadly useful computing tasks in computing devices, beginning from cell phones and winding up in net books. Each single center of an ARM Cortex A9 processor works as a superscalar out-of-order processor (see Figure 6) that implies, the processor consists of numerous parallel operable pipelines. Directions gotten in these pipelines can outpace each other with the goal that they can be finished in opposition to the request they are issued. The centers have a two-level cache system. Each L1 reserve can be designed from 16 to $64 \mathrm{~KB}$ that is very vast for an embedded processor. Utilizing such an expansive reserve underpins the outline for a high clock recurrence of $2 \mathrm{GHz}$ in order to accelerate the execution of a solitary string. Keeping in mind the end goal to keep up the coherency between the reserve substance and the memory, a communicate interconnect framework is utilized. Since the quantity of centers is still little, the hazard is low that the framework is running in bottlenecks. Two of such ARM Cortex A9 processors are incorporated with a C64x DSP (Digital Signal Processor) center and further controller centers in a heterogeneous multi-center framework on-chip solution called TI OMAP 4430. This system is proposed additionally as broadly useful processor for PDAs and mobile Internet devices (MIDs). Average information parallel applications do not favor as extremely effective for such processors. In this sense, the ARM Cortex A9 and the TI OMAP 4430 processors are more suited for errand parallel embedded applications. Contrary to those processors, the ECA (Elemental Computing Array) processor family focuses to low 
power handling of embedded data parallel assignments, e.g. in High Definition Video Processing or Software Defined Signal Conditioning. The design idea acknowledged in this arrangement is altogether different from the plans we find in the above depicted multi-center arrangements.

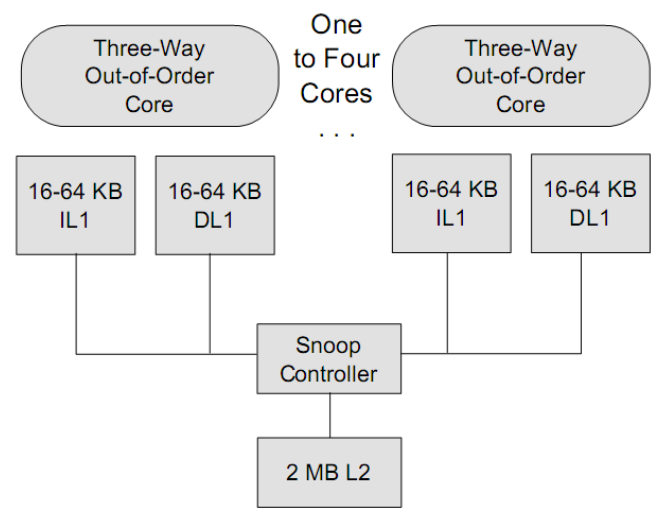

Fig. 6- Block diagram of the ARM Cortex-A9 MP, redrawn

Perhaps, it focuses toward a path likewise HPC frameworks will seek after later on. The heart of that design is a variety of fine-grain heterogeneous specific and programmable processor cores (see Figure 7). The embedded processor ECA64 comprises of four groups of such centers and each bunch totals one processor center working to RISC standards and further less difficult 15 ALUs which are custom-made to satisfy specific errands. The programming of that ALUs happens likewise as it is done in Field-Programmable Gate Arrays (FPGAs).

An important requirement for the low power qualities of the processors is the information driven operation method of the ALUs, i.e. the ALUs are just exchanged on if data is display at their information sources. Additionally the memory sub system is intended to bolster low power. All processor centers in one bunch share a local memory of $32 \mathrm{kB}$. The entrance to the local memory must be performed totally by software, which abstains from coordinating refined and power expending equipment control assets. This moves the many-sided quality of planning concurrent memory gets to the product. The interconnect is various leveled. Taking after the various leveled architecture organization of the processor centers likewise the interconnect framework must be organized progressively. Four processor centers are firmly coupled by means of a crossbar. In one bunch four of these crossbars associated centers are connected in an indicate point design utilizing a line framework. On the most elevated progressive level the four bunches are coupled through a bus and a bus administrator mediating the gets two of the groups on the bus. Hierarchically and heterogeneously composed processor, memory and interconnect frameworks, as we discover it in the ECA processor, are spearheading in our view for future embedded multi-center structures to accomplish both high computing performance and low power preparing. In any case, specific information parallelism applications require extra complex data access designs that consider the 2D or 3D nature of data streams given in such applications. Moreover, they should be very much custom fitted to a various leveled memory system to abuse the advantages such an organization offers.

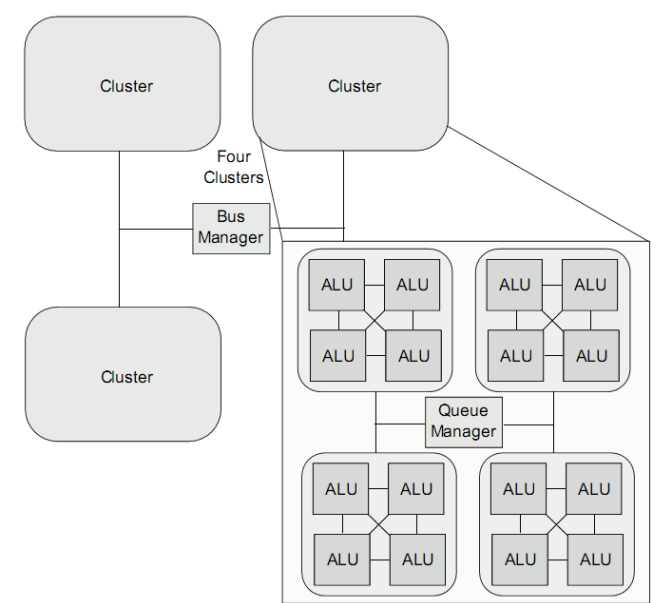

Fig. 7- Element CXI ECA-64 block diagram, redrawn

These are time covering of data processing and of information exchange to shroud inactivity and to expand transmission capacity by information buffering in pipelined architectures. 
Vol. 6, Issue 6, June 2017

\section{CONCLUSION}

In this paper, a coordinated parallelization and improvement technique has been developed, which can handle existing successive DSP codes written in C and produces advanced parallel code for multi-DSP target engineering. It consolidates a few diverse techniques at different stages in the arrangement chain. The thought of $\mathrm{C}$ as the overwhelming programming language for the usage of superior embedded systems and the advancement of a total streamlining and parallelization structure focusing on a class of far reaching business designs not just extends the logical understanding into compiler innovation, additionally gives significant learning to the inserted systems industry. Automatic optimization and parallelization for embedded systems in light of elite DSPs are presently doable. Significant impediments to abnormal state change and parallelization coming about because of poor programming style have been recognized and efficient techniques to defeat these issues have been created. Abnormal state changes to a great extent disregarded in the past have been appeared to be exceptionally effective with regards to DSP codes and an iterative input driven enhancement system has been proposed. A novel parallelization system involving information circulation and area advancements has been formulated and exactly assessed against pertinent DSP benchmarks. Important contributions to the improvement of novel arrangement procedures for elite embedded systems in light of single processors and in addition on numerous processors have been made. It is likely that we will see more research here as future Systems-On-Chip will contain bigger quantities of heterogeneous processors with non-standard memory designs, which will challenge existing compiler technology.

\section{REFERENCES}

[1] van Engelen, R. and Gallivan, K. (2001).An efficient algorithm for pointer-to-array access conversion for compiling and optimizing DSP applications.In Proceedings of International Workshop on Innovative Architecture (IWIA ’01), pages 80-89, Maui, HI, USA.

[2] Stephenson, M., Amarasinghe, S., Martin, M., and O'Reilly, U. (2002). Metaoptimization: Improving compiler heuristics with machine learning. Technical Report MIT-LCS-TM-634.

[3] Song, Y. and Lin, Y. (2000). Unroll-and-jam for imperfectly-nested loops in DSP applications. In Proceedings of the ACM International Conference on Compilers, Architectures, Synthesis for Embedded Systems (CASES '00), pages 148-156, San Jose, CA, USA.

[4] Qian, Y., Carr, S., and Sweany, P. (2002). Optimizing loop performance for clustered VLIW architectures. In Proceedings of the 11th IEEE International Conference on Parallel Architectures and Compiler Techniques (PACT '02), pages 271-280, Charlottesville, VA, USA.

[5] Paek, Y., Hoeflinger, J., and Padua, D. (2002). Efficient and precise array access analysis.ACM Transactions on Programming Languages and Systems, 24(1), 65- 109.

[6] O'Boyle, M. and Knijnenburg, P. (2002). Integrating loop and data transformations for global optimisation. Journal of Parallel and Distributed Computing, 62(4), 563-590.

[7] Numerix (2000). Numerix-DSP programming guidelines. http://www.numerixdsp.com/c coding.pdf.

[8] Mellor-Crummey, J., Adve, V., Broom, B., Chavarria-Miranda, D., Fowler, R., Jin, G., Kennedy, K., and Yi, Q. (2002). Advanced optimization strategies in the Rice dHPF compiler. Concurrency-Practice and Experience, 14(8-9), 741-767.

[9] Lorts, D. (2000). Combining parallelization techniques to increase adaptability and efficiency of multiprocessing DSP systems. In Proceedings of Ninth DSP Workshop (DSP 2000) - First Signal Processing Education Workshop (SPEd 2000), Hunt, TX, USA.

[10] Leupers, R. (2003). Offset assignment showdown: Evaluation of DSP address code optimization algorithms. In Proceedings of the 12th International Conference on Compiler Construction (CC '03), pages 290-302, Warsaw, Poland.

[11] Kondo, M., Fujita, M., and Nakamura, H. (2002). Software-controlled on-chip memory for high-performance and low-power computing. ACM Computer Architecture News, 30(3), 7-8. 